\title{
Stability and Instability of Relativistic
}

\section{Electrons in Classical Electromagnetic Fields}

\author{
Elliott H. Lieb \\ Departments of Mathematics and Physics \\ Princeton University \\ Princeton, NJ 08544-0708 \\ Heinz Siedentop \\ Jan Philip Solovej \\ Matematisk institutt Institut for matematiske fag \\ Universitetet i Oslo Aarhus universitet \\ Postboks 1053 \\ Ny Munkegade \\ N-0316 Oslo \\ DK-8000 Århus C \\ (C)1996 by the authors. Reproduction of this article, in its entirety, by any means is \\ permitted for non-commercial purposes
}




\title{
Dedicated to Bernard Jancovici on his 65th birthday.
}

\begin{abstract}
The stability of matter composed of electrons and static nuclei is investigated for a relativistic dynamics for the electrons given by a suitably projected Dirac operator and with Coulomb interactions. In addition there is an arbitrary classical magnetic field of finite energy. Despite the previously known facts that ordinary nonrelativistic matter with magnetic fields, or relativistic matter without magnetic fields is already unstable when $\alpha$, the fine structure constant, is too large it is noteworthy that the combination of the two is still stable provided the projection onto the positive energy states of the Dirac operator, which defines the electron, is chosen properly. A good choice is to include the magnetic field in the definition. A bad choice, which always leads to instability, is the usual one in which the positive energy states are defined by the free Dirac operator. Both assertions are proved here.
\end{abstract}

\section{Introduction}

The stability of matter concerns the many-electron and many-nucleus quantum mechanical problem and the question whether the ground state energy 
is finite (stability of the first kind). If so, is it bounded below by a constant (which is independent of the position of the nuclei) times the number of particles (stability of the second kind)? The linear lower bound is important for thermodynamics, which will not exist in the usual way without it.

The first positive resolution of this problem for the nonrelativistic Schrödinger equation was given by Dyson and Lenard [7, 8] and approached differently by Federbush [10]. The constant, i.e., the energy per particle, was considerably improved by Lieb and Thirring in [21, 22]. Following that, the stability of a relativistic version of the Schrödinger equation (in which $\mathbf{p}^{2}$ is replaced by $\sqrt{\mathbf{p}^{2}+m^{2}}$ ) was proved by Conlon [5] and later improved by Lieb and Yau [23] who showed that matter is stable in this model if and only if the fine structure constant $\alpha$ is small enough and if $Z \alpha \leq 2 / \pi$. (See [23] for a historical account up to 1995.) A recent result of Lieb, Loss, and Siedentop that we shall use is in [19] and is discussed in Section 3 .

In these works the nuclei are fixed in space because they are very massive and because we know that the nuclear motion is largely irrelevant for understanding matter. In other words, if nuclear motion were the only thing that prevented the instability of matter then the world would look very different from what it does. We continue this practice here. 
There is, however, a more important quantity that requires some attention, namely magnetic fields. It was noted that the action of such fields on the translational degrees of freedom of the electrons $\mathbf{p} \rightarrow \mathbf{p}+e \mathbf{A}$, can lower the energy only by an inconsequential amount. This is a kind of diamagnetic inequality. On the other hand, spin-magnetic field interaction (in which $(\mathbf{p}+e \mathbf{A})^{2}$ is replaced by the Pauli operator $[\boldsymbol{\sigma} \cdot(\mathbf{p}+e \mathbf{A})]^{2}=(\mathbf{p}+e \mathbf{A})^{2}+e \boldsymbol{\sigma} \cdot \mathbf{B}$ can cause instability. The energy is then unbounded below if arbitrarily large fields are allowed, but this is so only because the energy of the magnetic field has not been taken into account. Does the field energy, $(8 \pi)^{-1} \int B^{2}$, insure stability? This question was raised for the nonrelativistic case in [13] and finally settled in a satisfactory manner in [20] (see also Bugliaro et al. [4] and Fefferman [11]). The upshot of this investigation is that stability (of both first and second kinds) requires a bound on both $\alpha$ and on $Z \alpha^{2}$.

Other related results are the stability of non-relativistic matter with a second quantized, ultra-violet cut-off photon field (Fröhlich et al. 12]).

Both the passage to relativistic kinematics (which, in quantum mechanics, means that both the kinetic energy and the Coulomb potential scale with length in the same way, namely like an inverse length) and the introduction of the nonrelativistic Pauli operator require a bound on $\alpha$ and on $Z$ for 
stability. The combination of the two might be expected to lead to disaster. We find, however, that it does not necessarily do so!

Our main result is that matter is indeed stable with a suitably defined relativistic kinematics. This is shown in Section 3.

The proper way to introduce relativistic kinematics for spin- $1 / 2$ particles is via the Dirac operator, but this is unbounded below. A resolution of this problem, due to Dirac, is to permit the electrons to live only in the positive energy subspace of the Dirac operator. This idea was further pursued by Brown and Ravenhall [3] (see also Bethe and Salpeter in their Handbuch article [1]) to give a quantitative description of real atoms.

There are, however, other Dirac operators (which include electromagnetic potentials) whose positive subspace can be used to define the space in which the electrons can live. (To avoid confusion, let us note that the Hamiltonian is formally always the same and includes whatever fields happen to be present. The only point to be resolved is what part of the one-particle Hilbert space is allowed for electrons.) The review articles of Sucher [24, 25, 26] can be consulted here. These choices have also been used in quantum chemistry and other practical calculations, see, e.g., [14, [15].

All of these choices have in common that there is no creation of electron- 
positron pairs explaining the name "no-pair Hamiltonian" for the resulting energy operator. (Note that we could also treat positrons or a combination of electrons and positrons interacting by Coulomb forces in a similar way.)

There are three obvious choices to consider. One is the free Dirac operator. This always leads to instability of the first kind when a magnetic field is added unless the particle number is held to some small value (see Section (4). Note also that this choice leads to a non-gauge invariant model: multiplication of a state with the factor $\exp (i \phi(\mathbf{r}))$ for a non-constant gauge is not allowed, since it leads out of the positive spectral subspace.

Remarkably, the Dirac operator that includes the magnetic field always gives stability, if $Z$ and $\alpha$ are not too large, as in the two cases (relativistic without magnetic field and nonrelativistic with magnetic field) mentioned above (see Section 3). This model is gauge invariant.

The third choice which, indeed, is sometimes used, is to include both the one-body attractive electric potential of the nuclei and the magnetic field in the definition of the Dirac operator that defines the positive subspace. If this is done then the question of stability is immediately solved because the remaining terms in the Hamiltonian are positive, and hence the total energy is ipso facto positive. This choice, which is important but trivial in 
the context of this present inquiry, will not be mentioned further.

Oddly, the instability proof given in Subsection 4.2 is much more complicated than the stability proof (Section 3). This reverses the usual situation.

A preliminary version of this paper appeared in [27]; the present version is to be regarded as the original one (as stated in [27]) and contains several significant corrections to the preliminary text in [27]. In particular, the proof of Theorem 2 and the first half of the proof of Theorem 1 have been corrected and simplified.

\section{Basic Definitions}

The energy of $N$ relativistic electrons in the field of $K$ nuclei with atomic numbers $Z_{1}, \ldots, Z_{K} \in \mathbb{R}_{+}$located at $\mathbf{R}_{1}, \ldots, \mathbf{R}_{K} \in \mathbb{R}^{3}$ which are pairwise different in a magnetic field $\mathbf{B}=\nabla \times \mathbf{A}$ in the state $\Psi$ is - following the ideas of Brown and Ravenhall [3]-

$$
\mathcal{E}_{\mathcal{A}}[\Psi, \Psi]:=\left(\Psi,\left(\sum_{\nu=1}^{N} D_{\nu}(\mathbf{A})+\alpha V_{c}\right) \Psi\right)+\frac{1}{8 \pi} \int_{\mathbb{R}^{3}} B(\mathbf{r})^{2} d \mathbf{r}
$$


Here $D_{\nu}(\mathbf{A}):=\boldsymbol{\alpha} \cdot\left(-i \nabla_{\nu}+e \mathbf{A}\left(\mathbf{r}_{\nu}\right)\right)+m \beta$ is the Dirac operator with vector potential A. Furthermore,

$$
V_{c}:=-\sum_{\nu=1}^{N} \sum_{\kappa=1}^{K} \frac{Z_{\kappa}}{\left|\mathbf{r}_{\nu}-\mathbf{R}_{\kappa}\right|}+\sum_{\substack{\mu, \nu=1 \\ \mu<\nu}}^{N} \frac{1}{\left|\mathbf{r}_{\mu}-\mathbf{r}_{\nu}\right|}+\sum_{\substack{\kappa, \lambda=1 \\ \kappa<\lambda}}^{K} \frac{Z_{\kappa} Z_{\lambda}}{\left|\mathbf{R}_{\kappa}-\mathbf{R}_{\lambda}\right|}
$$

is the Coulomb interaction between the particles, and $B(\mathbf{r}):=|\nabla \times \mathbf{A}(\mathbf{r})|$ is the modulus of the magnetic field. Planck's constant divided by $2 \pi$ and the velocity of light, are taken to be one in suitable units. The fine structure constant $\alpha$ equals $e^{2}$, where $-e$ is the electron charge. Experimentally, $\alpha$ is about $1 / 137.037$. The mass of the electron is denoted by $m$. The $4 \times 4$ matrices $\boldsymbol{\alpha}$ and $\beta$ are the four Dirac matrices in standard representation, namely

$$
\boldsymbol{\alpha}=\left(\begin{array}{cc}
0 & \boldsymbol{\sigma} \\
\boldsymbol{\sigma} & 0
\end{array}\right), \sigma_{1}=\left(\begin{array}{cc}
0 & 1 \\
1 & 0
\end{array}\right), \sigma_{2}=\left(\begin{array}{cc}
0 & -i \\
i & 0
\end{array}\right), \sigma_{3}=\left(\begin{array}{cc}
1 & 0 \\
0 & 1
\end{array}\right),
$$

and

$$
\beta=\left(\begin{array}{cccc}
1 & 0 & 0 & 0 \\
0 & 1 & 0 & 0 \\
0 & 0 & -1 & 0 \\
0 & 0 & 0 & -1
\end{array}\right) .
$$

Finally, the state $\Psi$ should have finite kinetic energy, i.e., it should be in the Sobolev space $H^{1 / 2}\left[\left(\mathbb{R}^{3} \times\{1,2,3,4\}\right)^{N}\right]$, and should also be in the 
electronic Hilbert space of antisymmetric spinors

$$
\mathcal{H}_{N, \mathcal{A}}:=\bigwedge_{\nu=1}^{N} \mathcal{H}_{+}
$$

where $\mathcal{H}_{+}$is the positive spectral subspace of the Dirac operator $D(\mathcal{A})$ and where $\mathcal{A}$ is some vector potential to be chosen later. The vector potential $\mathcal{A}$ serves to define the positive subspace. Two choices will be considered here. One is $\mathcal{A}=\mathbf{0}$, in which case we are talking about the free Dirac operator. This choice, or model, goes back to Brown and Ravenhall [3]. As we shall see in Section 4 , the resulting energy functional-apart from being not gauge invariant - is not bounded from below. A natural modification of the model, namely to take $\mathcal{A}:=\mathbf{A}$ is not only gauge invariant, but will also turn out to be stable of the second kind (see Section 3).

The quantity of interest is the lowest possible energy

$$
E_{N, K}:=\inf \mathcal{E}_{\mathcal{A}}
$$

where the infimum is taken over all allowed states $\Psi$, all allowed vector potentials $\mathbf{A}$, and over all pairwise different nuclear positions $\mathbf{R}_{1}, \ldots, \mathbf{R}_{K}$.

In the case of a single nucleus without a magnetic field, the energy form $\mathcal{E}_{\mathbf{0}}$ was shown in [9] to be bounded from below, if and only if $\alpha Z \leq \alpha Z_{C}:=$ $2 /(\pi / 2+2 / \pi)>2 / \pi$, which corresponds to $Z \approx 124$. We will not be able 
to reach this value in the general case of many nuclei and when the electron state space is not determined by the free Dirac operator. The reason is that special techniques were used in [9] to handle the one-nucleus case; these techniques took advantage of the weakening of the Coulomb singularities caused by the fact that states in $\mathcal{H}_{+}$cannot be localized in space arbitrarily sharply. Unfortunately, we do not know how to implement this observation with magnetic fields and many nuclei.

\section{$3 \quad$ Stability with the Modified Projector}

Our proof of the stability of matter when the vector potential $\mathbf{A}$ is included in the definition of the positive energy electron states will depend essentially on three inequalities:

BKS inequality: For any self-adjoint operator $X$, the negative (positive) part, $X_{\mp}$ is defined to be $(|X| \mp X) / 2$. Given two non-negative selfadjoint operators $C$ and $D$ such that $\left(C^{2}-D^{2}\right)_{-}^{1 / 2}$ is trace class, we have the trace inequality

$$
\operatorname{tr}(C-D)_{-} \leq \operatorname{tr}\left(C^{2}-D^{2}\right)_{-}^{1 / 2}
$$

This is a special case of a more general inequality of Birman, Koplienko, 
and Solomyak [2]; in particular, the number 2 in (四) can be replaced by any $p>1$. A proof for the special case of the inequality needed here is

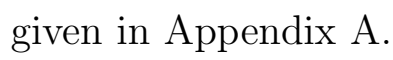

Stability of relativistic matter: On $\bigwedge_{\nu=1}^{N}\left(H^{1 / 2}\left(\mathbb{R}^{3}\right) \otimes \mathbb{C}^{q}\right)$, the fermionic Hilbert space, we have

$$
\sum_{\nu=1}^{N}\left|-i \nabla_{\nu}-\mathcal{A}\right|+\tilde{\alpha} V_{c} \geq 0
$$

(where $|\cdots|$ means $\sqrt{(\cdots)^{2}}$ ) for all vector fields $\mathcal{A}: \mathbb{R}^{3} \rightarrow \mathbb{R}^{3}$ with, e.g., square integrable gradients, if

$$
1 / \tilde{\alpha} \geq 1 / \tilde{\alpha}_{c}:=(\pi / 2) Z+2.2159 q^{1 / 3} Z^{2 / 3}+1.0307 q^{1 / 3}
$$

and $Z_{1}, \ldots, Z_{K} \leq Z$.

We wish to use this inequality for 4 -component spinors, i.e., $q=4$. However, we are interested in the subspace $\mathcal{H}_{N, \mathcal{A}}$ in which the particles are restricted to the positive energy subspace of the Dirac operator, $D(\mathcal{A})$. Although $q=4$, the 'effective' $q$ is really 2 , and the analysis in Appendix B is our justification for this. The only thing that really counts in deriving (5) is the bound on the reduced one-body density matrix $\gamma$ mentioned in Appendix B. 
The stability of the relativistic Hamiltonian (可) was first shown by Conlon [5] for $\mathcal{A}=\mathbf{0}$. The best currently available constants with $\mathcal{A}=\mathbf{0}$ are in 23] while (6), which is taken from [19], is the best known result for general $\mathcal{A}$.

Semi-classical bound: Given a positive constant $\mu$, a real vector field $\mathcal{A}$ with, e..g., square integrable gradients, and a real-valued function $\varphi \in$ $L^{2}\left(\mathbb{R}^{3}\right)$ the inequality

$$
\operatorname{tr}\left[(-i \mu \nabla-\mathcal{A})^{2}-\varphi\right]_{-}^{1 / 2} \leq \frac{L_{1 / 2,3}}{\mu^{3}} \int_{\mathbb{R}^{3}} \varphi_{+}^{2}
$$

holds, which is a special case of the Lieb-Thirring inequality (see 22, [17]). It is known that $L_{1 / 2,3} \leq 0.06003$. The left side of (7) is simply $\sum_{j}\left|\lambda_{j}\right|^{1 / 2}$, where the $\lambda_{j}$ are the negative eigenvalues of the operator $[\cdots]$.

As an illustration of the usefulness of the trace estimate (4), let us combine it with the Lieb-Thirring inequality (7) (or any other Lieb-Thirring inequality for that matter) to derive some previously known inequalities. The constants obtainable in this way are comparable with the best ones known so far:

Daubechies inequality: We begin with a "relativistic" inequality that was first proven by Daubechies [6]. By replacing $\varphi$ by $\varphi^{2}$ in (7), we get using 
(4)

$$
\operatorname{tr}(|-i \nabla-\mathcal{A}|-\varphi)_{-} \leq L_{1 / 2,3} \int_{\mathbb{R}^{3}} \varphi_{+}^{4}
$$

The constant 0.06003 obtained here should be compared with the number 0.0258 in 6 .

Non-relativistic magnetic stability: A non-relativistic analogue of our main problem is to bound the form

$$
\tilde{\mathcal{E}}:=\left(\Psi,\left(\sum_{\nu=1}^{N} P_{\nu}(\mathbf{A})+\alpha V_{c}\right) \Psi\right)+\frac{1}{8 \pi} \int_{\mathbb{R}^{3}} B(\mathbf{r})^{2} d \mathbf{r}
$$

which was treated in 20]. Here $P_{\nu}(\mathbf{A}):=\left[\boldsymbol{\sigma} \cdot\left(-i \nabla_{\nu}+e \mathbf{A}\left(\mathbf{r}_{\nu}\right)\right)\right]^{2}$ is the Pauli operator with vector potential A.

First, we note that $x^{2} \geq+\lambda|x|-\lambda^{2} / 4$ holds. A constant in the energy form, however, is irrelevant for checking on stability of the second kind. Using (5) it is then enough to show the positivity of

$$
-\operatorname{tr}\left(\lambda P(\mathbf{A})^{1 / 2}-\kappa|-i \nabla+e \mathbf{A}|\right)_{-}+\frac{1}{8 \pi} \int_{\mathbb{R}^{3}} B(\mathbf{r})^{2} d \mathbf{r}
$$

where we have set $\kappa:=\alpha / \tilde{\alpha}_{c}$. The trace in this and the next expression are over $L^{2}\left(\mathbb{R}^{3}\right) \otimes \mathbb{C}^{2}$. Using the BKS inequality gives the lower bound

$$
\left.-\operatorname{tr}\left[\left(\lambda^{2}-\kappa^{2}\right)|-i \nabla+e \mathbf{A}|^{2}\right)-e \lambda^{2} B(\mathbf{r})\right]_{-}^{1 / 2}+\frac{1}{8 \pi} \int_{\mathbb{R}^{3}} B(\mathbf{r})^{2} d \mathbf{r}
$$


Applying the Lieb-Thirring inequality (7) yields the following sufficient condition for stability (recall that $\alpha=e^{2}$ and that there are two spin states)

$$
2 L_{\frac{1}{2}, 3} \frac{\lambda^{4} \alpha}{\left(\lambda^{2}-\kappa^{2}\right)^{3 / 2}} \leq \frac{1}{8 \pi} .
$$

Optimizing in $\lambda$ gives

$$
2 L_{\frac{1}{2}, 3} \frac{16 \alpha^{2}}{3^{3 / 2} \tilde{\alpha}_{c}} \leq \frac{1}{8 \pi},
$$

which gives for the physical values $\alpha \approx 1 / 137.037$ and $q=2$ a range of stability up to $Z \leq 1096$, which is to be compared with $Z \leq 1050$ in [20].

We turn now to our main result.

Theorem 1 Let $Z_{1}, \ldots, Z_{K} \leq Z<2 /(\pi \alpha)$ and let $\alpha \leq \alpha_{c}$ where $\alpha_{c}$ is the unique solution of the equation

$$
\left(16 \pi L_{\frac{1}{2}, 3} \alpha_{c}\right)^{2 / 3}=1-\alpha_{c}^{2} / \tilde{\alpha}_{c}^{2},
$$

with $\tilde{\alpha}_{c}:=\left[(\pi / 2) Z+2.2159 \cdot 2^{1 / 3} Z^{2 / 3}+1.0307 \cdot 2^{1 / 3}\right]^{-1}$ as in (6). Then $\mathcal{E}_{\mathbf{A}}$ is non-negative.

Numerically, this gives

$$
Z \leq 56
$$


when evaluated with the experimental value $\alpha \approx 1 / 137.037$ for the fine structure constant. Alternatively, considering hydrogen only, i.e., $Z=1$, we obtain the upper bound

$$
\alpha \leq 1 / 8.139
$$

for the fine structure constant. It is a challenge to improve this result so that it covers all physical nuclear charges and the physical value of the fine structure constant, as was done for $K=N=1$ and $\mathbf{A}=\mathbf{0}$ in [9].

Proof. The first step in our proof is to utilize (5) to replace $V_{c}$ by the one-body operator $\left(-1 / \tilde{\alpha}_{c}\right) \sum_{\nu=1}^{N}\left|-i \nabla_{\nu}+e \mathbf{A}\right|$, where $\tilde{\alpha}_{c}$ is given by (6) with $q=2$, as we explained just after (6). (The idea of using the relativistic stability result (5) to bound the Coulomb potential by a one-body operator first appears in [20].) Our energy $\mathcal{E}_{\mathbf{A}}$ is now bounded below by

$$
\mathcal{E}^{\prime}(\Psi):=\left(\Psi, \sum_{\nu=1}^{N}\left(D_{\nu}(\mathbf{A})-\kappa\left|-i \nabla_{\nu}+e \mathbf{A}\left(\mathbf{r}_{\nu}\right)\right|\right) \Psi\right)+\frac{1}{8 \pi} \int_{\mathbb{R}^{3}} B(\mathbf{r})^{2} d \mathbf{r},
$$

where $\kappa:=\alpha / \tilde{\alpha}_{c}$.

The first term on the right side of (11) is bounded below by the sum of the negative eigenvalues, $-\operatorname{tr} h_{-}$, of the one-body operator

$$
h:=\Lambda^{+}(D(\mathbf{A})-\kappa|-i \nabla+e \mathbf{A}(\mathbf{r})|) \Lambda^{+},
$$

where $\Lambda^{+}$is the projector onto the positive spectral subspace of $D(\mathbf{A})$. 
Let us define

$$
S:=|D(A)|-\kappa|-i \nabla+e \mathbf{A}(\mathbf{r})|,
$$

whence $h=\Lambda_{+} S \Lambda_{+}$, because $\Lambda_{+} D(\mathbf{A}) \Lambda_{+}=\Lambda_{+}|D(\mathbf{A})| \Lambda_{+}$. We note that for any two self-adjoint operators $X$ and $\rho$ with $X \geq 0$ and $0 \leq \rho \leq 1$, $\operatorname{tr} X \geq \operatorname{tr} \rho X$. With $\rho$ taken to be the projector onto the negative spectral subspace of $h$ we then have that

$$
\begin{aligned}
\operatorname{tr} h_{-} & =-\operatorname{tr} \rho h=-\operatorname{tr} \rho \Lambda_{+} S \Lambda_{+} \\
& =\operatorname{tr} \rho \Lambda_{+} S_{-} \Lambda_{+}-\operatorname{tr} \rho \Lambda_{+} S_{+} \Lambda_{+} \\
& \leq \operatorname{tr} \Lambda_{+} S_{-} \Lambda_{+} .
\end{aligned}
$$

We introduce the $4 \times 4$ unitary

$$
U=\left(\begin{array}{cc}
0 & 1 \\
-1 & 0
\end{array}\right)
$$

and note that $U^{-1} D(\mathbf{A}) U=-D(\mathbf{A})$. Therefore, $U^{-1} \Lambda_{+} U=\Lambda_{-}$.

It follows from the spectral theorem that for any self-adjoint $X$, unitary $U$, and function $F$

$$
F\left(U^{-1} X U\right)=U^{-1} F(X) U .
$$

With $F(t)=|t|$, we then have that $U^{-1}|D(\mathbf{A})| U=|D(\mathbf{A})|$, and hence $U^{-1} S U=S$. 
Therefore, since $U^{-1} \Lambda_{+} U=\Lambda_{-}$, and with $F(t)=\frac{1}{2}(|t|-t)=t_{-}$, we have that $U^{-1} S_{-} U=S_{-}$and

$$
\operatorname{tr} \Lambda_{+} S_{-} \Lambda_{+}=\operatorname{tr} \Lambda_{-} S_{-} \Lambda_{-} .
$$

Hence, using (12),

$$
\operatorname{tr} h_{-} \leq \frac{1}{2} \operatorname{tr}\left(\Lambda_{+} S_{-} \Lambda_{+}\right)+\frac{1}{2} \operatorname{tr} \Lambda_{-} S_{-} \Lambda_{-}=\frac{1}{2} \operatorname{tr} S_{-} .
$$

(Note: much of the preceding discussion was needed only to get the factor $1 / 2$ here. This factor improves our final constants for stability.)

Next, we use the BKS inequality (四) to bound $\operatorname{tr} S_{-}$as follows:

$$
\begin{gathered}
\operatorname{tr} h_{-} \leq \frac{1}{2} \operatorname{tr} S_{-} \leq \frac{1}{2} \operatorname{tr}\left[D(\mathbf{A})^{2}-\kappa^{2}|-i \nabla+e \mathbf{A}(\mathbf{r})|^{2}\right]_{-}^{1 / 2} \\
\text { However, } D(\mathbf{A})^{2}=\left(\begin{array}{rr}
Y & 0 \\
0 & Y
\end{array}\right) \text { with } Y=P(\mathbf{A})+m^{2} \text {, and where } \\
P(\mathbf{A})=[\boldsymbol{\sigma} \cdot(-i \nabla+e \mathbf{A})]^{2}=|-i \nabla+e \mathbf{A}(\mathbf{r})|^{2}+e \boldsymbol{\sigma} \cdot \mathbf{B}(\mathbf{r})
\end{gathered}
$$

is the Pauli operator.

Since $X \mapsto \operatorname{tr} X_{-}^{1 / 2}$ is operator monotone decreasing, we see that our lower bound for the energy is monotone increasing in $m$, and thus it suffices to prove the positivity of $\mathcal{H}_{N, \mathbf{A}}$ in the massless case. The key observation is 
that our lower bound involves only $\operatorname{tr} S_{-}$in the entire one-body space, not the positive energy subspace. The energy would not be obviously monotone in $m$ if we had to restrict functions to the positive subspace, since changing $m$ would also entail changing the space. This problem does not arise in the absence of the positive subspace constraint.

Because of the 'diagonal' structure of the operator $S$, we can drop the factor $1 / 2$ by replacing the trace on $L^{2}\left(\mathbb{R}^{3}\right) \otimes \mathbb{C}^{4}$ by the trace on $L^{2}\left(\mathbb{R}^{3}\right) \otimes \mathbb{C}^{2}$. This yields

$$
\begin{aligned}
& \mathcal{E}_{\mathbf{A}}[\Psi, \Psi] \geq-\operatorname{tr}\left[P(\mathbf{A})-\kappa^{2}(-i \nabla+e \mathbf{A})^{2}\right]_{-}^{1 / 2}+\frac{1}{8 \pi} \int_{\mathbb{R}^{3}} B(\mathbf{r})^{2} d \mathbf{r} \\
\geq & -2 \operatorname{tr}\left[\left(1-\kappa^{2}\right)(-i \nabla+e \mathbf{A})^{2}-e B\right]_{-}^{1 / 2}+\frac{1}{8 \pi} \int_{\mathbb{R}^{3}} B(\mathbf{r})^{2} d \mathbf{r} .
\end{aligned}
$$

We regard the operator in the second line as acting on functions (of one component only) instead of spinors, which accounts for the factor two (and not one).. Finally we apply the Lieb-Thirring inequality (7) to the right hand side yielding (recall that $e^{2}=\alpha$ )

$$
\mathcal{E}_{\mathbf{A}}[\Psi, \Psi] \geq\left[-2 L_{\frac{1}{2}, 3} \alpha\left(1-\alpha^{2} / \tilde{\alpha}_{c}^{2}\right)^{-3 / 2}+1 /(8 \pi)\right] \int_{\mathbb{R}^{3}} B^{2}(\mathbf{r})^{2} d \mathbf{r}
$$

Thus we need

$$
\left(16 \pi L_{\frac{1}{2}, 3} \alpha\right)^{2 / 3} \leq 1-\alpha^{2} / \tilde{\alpha}_{c}^{2}
$$


Since the right hand side of this inequality is monotone decreasing in $\alpha$ for positive $\alpha$, while the left hand side is monotone increasing, there is a unique $\alpha_{c}$ for which equality holds in (15). Inserting the value (6) with $q=2$ for $\tilde{\alpha}_{c}$ yields - together with the second requirement on $Z_{1}, \ldots, Z_{K}$ in the relativistic bound - the claimed stability criterion.

\section{Instability with the Free Dirac Operator}

In this section we shall discuss the Brown-Ravenhall model ([3]). That is to say we consider the energy expression (1) with zero vector potential in the definition of the allowed electronic states (3), i.e., we take only $\Psi \in$ $\mathcal{H}_{N, \mathbf{0}}=\bigwedge^{N} \mathcal{H}_{+}$, where $\mathcal{H}_{+}$is the positive spectral subspace of the operator $-\boldsymbol{\alpha} \cdot i \nabla+m \beta$. We shall prove that there is no stability is in this model by showing that for any (sufficiently large) particle number, $N$, and any $\alpha>0$ the energy is unbounded below. In other words, "stability of the first kind" is violated. It is nevertheless true, however, that for any choice of particle numbers and nuclear charges there is always a sufficiently small, nonzero $\alpha$ such that the energy is bounded below by zero.

Since the positive spectral subspace $\mathcal{H}_{+}$for the free Dirac operator is 
not invariant under gauge transformations we see that this Brown-Ravenhall model is not gauge invariant. (The previous, modified model discussed in Section 5 is not only stable, it is also gauge invariant.) More precisely, the energy spectrum depends not only on $\mathbf{B}$ but in fact on the full gauge potential A. The Brown-Ravenhall model is therefore physically meaningfully defined only if we make a fixed choice of gauge. The natural choice is the Coulomb gauge (radiation gauge),

$$
\nabla \cdot \mathbf{A}=0
$$

since in quantum electro-dynamics this gauge implies that electrons interact via the usual Coulomb potentials and the coupling to the transverse field is minimal, i.e., derivatives are replaced by covariant derivatives.

The interesting quantity is the lowest energy that the system can have.

\section{Definition 1 (Energy)}

$$
E_{N, K}:=\inf \mathcal{E}_{0}[\Psi, \Psi] .
$$

where the infimum is taken over all divergence free $\mathbf{A}$ fields, pairwise distinct nuclear locations $\mathbf{R}_{1}, \ldots, \mathbf{R}_{K}$ and normalized, antisymmetric states $\Psi \in \mathcal{H}_{N, \mathbf{0}}$. 


\subsection{Stability with Small $\alpha$ and Small Particle Number}

Since this result is not a main point of this paper we shall be brief - even sketchy. If a single particle $\Psi$ is in the positive spectral subspace of $D(\mathbf{0})$ then the action of $D(\mathbf{0})$ on $\Psi$ is the same as multiplication of each component by $\left(p^{2}+m^{2}\right)^{1 / 2}$ in Fourier space. For such functions we see that $(\Psi, D(\mathbf{0}) \Psi)$ exceeds $(\Psi,|\nabla| \Psi)$, so we may as well replace $D(\mathbf{0})$ by $|\nabla|$ and also drop the condition that $\Psi$ belong to the positive spectral subspace of $D(\mathbf{0})$.

The next step is to use the lower bound on $V_{c}$ in (5) so that the energy is now bounded below by a sum of one-body operators, in a manner similar to that in Section 3 with $\tilde{\alpha}_{c}$ as in Theorem 1):

$$
\mathcal{E}_{\mathbf{0}}[\Psi, \Psi] \geq\left(\Psi, \sum_{\nu=1}^{N}\left(1-\frac{\alpha}{\tilde{\alpha}_{c}}\right)\left|\nabla_{\nu}\right| \Psi\right)+\sqrt{\alpha} \int_{\mathbb{R}^{3}} \mathbf{j} \cdot \mathbf{A}+\frac{1}{8 \pi} \int_{\mathbb{R}^{3}} B^{2}
$$

(Note again that the 'effective spin' $q$ is 2 , as can be seen be repeating the above argument.) Here $\mathbf{j}(\mathbf{r})$ is the current in the state $\Psi$ and it is trivially bounded above pointwise by the density $\rho(\mathbf{r})$ in the state $\Psi$ (defined in Appendix B]). Therefore the integral involving $\mathbf{A}$ is bounded below by

$$
\int_{\mathbb{R}^{3}} \mathbf{j} \cdot \mathbf{A} \geq-\int_{\mathbb{R}^{3}} \rho A \geq-\|A\|_{6} N^{1 / 3}\|\rho\|_{4 / 3}^{2 / 3}
$$


Now $\int B^{2} \geq \int|\nabla A|^{2}$ and this is not less than $K_{3}^{-2}\|A\|_{6}^{2}$ by Sobolev's inequality where $K_{3}=4^{1 / 3}(3 \pi)^{-1 / 2} \pi^{-1 / 6}$ (see [16], p. 367). Similarly, the kinetic en-

$\operatorname{ergy}\left(\Psi, \sum_{\nu=1}^{N}\left|\nabla_{\nu}\right| \Psi\right)$ is bounded below by $1.63 q^{-1 / 3} \int \rho^{4 / 3}$, which was proved by Daubechies [6] and which follows from (8). If we use these inequalities and then minimize the energy with respect to the unknown quantity $\|A\|_{6}$, we easily find that the energy is non-negative as long as

$$
1.63\left(1-\alpha / \tilde{\alpha}_{c}\right) \geq 2 \pi N^{2 / 3} K_{3}^{2} q^{1 / 3} \alpha
$$

with $q=2$.

We shall show in Subsection 4.2 that the condition that $N^{2 / 3} \alpha$ is small, which - as we just proved - ensures boundedness from below, is in fact also necessary for the energy to be bounded from below.

\subsection{Instability for All $\alpha$ and Large Particle Number}

The main result of this section is that there is no stability in this model for any fixed, positive $\alpha$ if $N$ and $K$ are allowed to be arbitrary.

Theorem 2 (Instability) There exists a universal number $C>0$ such that for all values of the parameters $\alpha>0, m \geq 0, K=1,2,3, \ldots$, and all values 
of $N=1,2,3, \ldots$, and of $Z_{1}, Z_{2}, \ldots, Z_{K}$ satisfying

$$
\sum_{\kappa=1}^{K} Z_{\kappa}>C \max \left\{\alpha^{-3 / 2}, 1\right\}, \quad N>C \max \left\{\alpha^{-3 / 2}, 1\right\}, \quad \sum_{\kappa=1}^{K} Z_{\kappa}^{2}>2
$$

we have

$$
E_{N, K}=-\infty
$$

Proof. The theorem follows if, for all $E>0$, we show the existence of three quantities for which $\mathcal{E}_{\mathbf{0}}[\Psi, \Psi] \leq-E$, with $\Psi=\psi_{1} \wedge \cdots \wedge \psi_{N}$ :

A. A vector potential $\mathbf{A}$ with $\nabla \cdot \mathbf{A}=0$.

B. Orthonormal spinors $\psi_{1}, \ldots, \psi_{N} \in \mathcal{H}_{+}$.

C. Nuclear coordinates $\mathbf{R}_{1}, \ldots, \mathbf{R}_{K}$.

Our construction will depend on four parameters (to be specified at the very end), $\delta>0$ a momentum scale, which we shall let tend to infinity, $\theta>0$, which will be chosen sufficiently small (but independently of $N$ ), and $P, A_{0}>0$ which will be chosen as functions of $N$. Finally we denote by $\mathbf{n}_{1}, \mathbf{n}_{2}, \mathbf{n}_{3}$ the coordinate vectors $(1,0,0),(0,1,0),(0,0,1)$ respectively. We shall use the notation that $\boldsymbol{\omega}_{p}=\mathbf{p} /|\mathbf{p}|$ is the unit vector in the direction $\mathbf{p} \in \mathbb{R}^{3}$ 
A. The vector potential. We choose the vector potential A to have Fourier transform

$$
\hat{\mathbf{A}}(\mathbf{p}):=A_{0} \chi_{B(0,5 \delta)}(\mathbf{p})\left(\mathbf{n}_{2} \cdot \boldsymbol{\omega}_{\mathbf{p}}\right) \mathbf{n}_{3} \times \boldsymbol{\omega}_{\mathbf{p}}
$$

where $\chi_{B(0,5 \delta)}$ denotes the characteristic function (in $\mathbf{p}$-space) of the ball $B(0,5 \delta)$ centered at 0 with radius $5 \delta$. Note first that $\mathbf{A}$ is real since $\hat{\mathbf{A}}$ is real and $\hat{\mathbf{A}}(\mathbf{p})=\hat{\mathbf{A}}(-\mathbf{p})$. Moreover, $\mathbf{A}$ is divergence free, i.e., it is in the Coulomb gauge, since $-i \widehat{\nabla \cdot \mathbf{A}}(\mathbf{p})=\mathbf{p} \cdot \hat{\mathbf{A}}(\mathbf{p})=0$. We easily estimate the self-energy of the magnetic field $\mathbf{B}=\nabla \times \mathbf{A}$ corresponding to $\mathbf{A}$

$$
\frac{1}{8 \pi} \int(\nabla \times \mathbf{A})^{2}=\frac{1}{8 \pi} \int_{\mathbb{R}^{3}}|\mathbf{p} \times \hat{\mathbf{A}}(\mathbf{p})|^{2} d \mathbf{p} \leq A_{0}^{2} 2^{-1} \int_{0}^{5 \delta} p^{4} d p=2^{-1} 5^{4} A_{0}^{2} \delta^{5}
$$

Finally, we note for later use that

$$
\hat{\mathbf{A}}(\mathbf{p}) \cdot \mathbf{n}_{1}=-A_{0} \chi_{B(0,5 \delta)}(\mathbf{p})\left(\mathbf{n}_{2} \cdot \boldsymbol{\omega}_{\mathbf{p}}\right)^{2}
$$

B. The orthonormal spinors. For $\mathbf{p}_{0} \in \mathbb{R}^{3}$ define

$$
u_{\mathbf{p}_{0}}(\mathbf{p})=\sqrt{3 /(4 \pi)} \delta^{-3 / 2}\left(\begin{array}{c}
\chi_{B(0, \delta)}\left(\mathbf{p}-\mathbf{p}_{0}\right) \\
0
\end{array}\right)
$$

We then have a normalized $\psi_{\mathbf{p}_{0}} \in \mathcal{H}_{+}$given by

$$
\widehat{\psi_{\mathbf{p}_{0}}}(\mathbf{p})=(2 E(p)(E(p)+E(0)))^{-1 / 2}\left(\begin{array}{c}
(E(p)+E(0)) u_{\mathbf{p}_{0}}(\mathbf{p}) \\
\mathbf{p} \cdot \boldsymbol{\sigma} u_{\mathbf{p}_{0}}(\mathbf{p})
\end{array}\right)
$$


where $E(p)=\left(p^{2}+m^{2}\right)^{1 / 2}$. Recall that this is the general form of a spinor in the positive spectral subspace $\mathcal{H}_{+}$for the free Dirac operator.

For the sake of simplicity we shall henceforth assume that $m=0$. We leave it to the interested reader to check the estimates for the general case $m \neq 0$. We shall indeed consider spinors with momenta $p$ such that we have $p^{2}\left(m^{2}\right)^{-1} \rightarrow \infty$ as $\delta \rightarrow \infty$, i.e., $E(p) \approx p$. It is therefore straightforward to estimate the expressions in the general case $m \neq 0$ by the corresponding expressions for $m=0$.

In particular, we have, for $m=0$,

$$
\widehat{\psi_{\mathbf{p}_{0}}}(\mathbf{p})=2^{-1 / 2}\left(\begin{array}{c}
u_{\mathbf{p}_{0}}(\mathbf{p}) \\
\boldsymbol{\omega}_{\mathbf{p}} \cdot \boldsymbol{\sigma} u_{\mathbf{p}_{0}}(\mathbf{p})
\end{array}\right)
$$

We shall choose $N$ points $\mathbf{p}_{1}, \ldots, \mathbf{p}_{N} \in \mathbb{R}^{3}$ such that the following conditions are satisfied.

1. $\min _{\nu \neq \mu}\left|\mathbf{p}_{\nu}-\mathbf{p}_{\mu}\right|>2 \delta$

2. $P \leq p_{\nu} \leq 2 P$, for all $\nu=1, \ldots, N$

3. $\boldsymbol{\omega}_{\mathbf{p}_{\nu}} \cdot \mathbf{n}_{1} \geq 1-\theta^{2}$, for all $\nu=1, \ldots, N$

Condition 1 ensures that the spinors $\psi_{\mathbf{p}_{1}}, \ldots, \psi_{\mathbf{p}_{N}}$ are orthonormal. The importance of Conditions 2 and 3 will hopefully become clear below. 
In order that Conditions 1, 2 and 3 are consistent with having $N$ points (for large $N$ ) we must ensure that $N$ balls of radius $\delta$ can be packed into the domain defined by Condtions 2 and 0 . Since small enough balls can fill at least half the volume of the given region we simply choose $P$ such that

$$
2 N \leq \frac{\operatorname{Vol}\left(\left\{\mathbf{p} \mid P \leq p \leq 2 P, 1-\theta^{2} \leq \boldsymbol{\omega}_{\mathbf{p}} \cdot \mathbf{n}_{1}\right\}\right)}{(4 \pi / 3) \delta^{3}}=\frac{7}{2} \theta^{2} \frac{P^{3}}{\delta^{3}}
$$

Note that the assumption that $N$ is larger than some universal number ensures that the balls are small, i.e., that $\delta$ is small enough compared to $P$. Thus, we have the condition

$$
P \geq\left(\frac{4 N}{7 \theta^{2}}\right)^{1 / 3} \delta
$$

In particular, since we shall choose $\theta$ independently of $N$ we may assume that $N$ is large enough that the above condition implies $P \theta \geq 2 \delta$. (Since we shall choose $\delta \rightarrow \infty$ we see that the momenta of the spinors satisfy $p^{2} m^{-2} \rightarrow \infty$.)

We are now prepared to calculate $\left(\Psi, \sum_{\nu=1}^{N} D_{\nu}(\mathbf{A}) \Psi\right)$, where $\Psi=\psi_{\mathbf{p}_{1}} \wedge$ $\cdots \wedge \psi_{\mathbf{p}_{N}}$. We obtain

$$
\left(\Psi, \sum_{\nu=1}^{N} D_{\nu}(\mathbf{A}) \Psi\right)=\sum_{\nu=1}^{N}\left(T_{\nu}+\int e \mathbf{j}_{\nu} \cdot \mathbf{A}\right)
$$

where

$$
\mathbf{j}_{\nu}(\mathbf{r}):=\psi_{\mathbf{p}_{\nu}}^{*}(\mathbf{r}) \boldsymbol{\alpha} \psi_{\mathbf{p}_{\nu}}(\mathbf{r})
$$


is the current of the $\nu$-th one-electron state $\psi_{\mathbf{p}_{\nu}}$, and

$$
T_{\nu}:=\left(\psi_{\mathbf{p}_{\nu}},(-i \boldsymbol{\alpha} \cdot \nabla+\beta m) \psi_{\mathbf{p}_{\nu}}\right)=\int E(p)\left|u_{\mathbf{p}_{\nu}}(\mathbf{p})\right|^{2} d \mathbf{p} \leq p_{\nu}+\delta,
$$

since we have assumed that $m=0$ and hence $E(p)=p$.

We must evaluate the current integral

$$
\begin{aligned}
\int \mathbf{j}_{\nu} \cdot \mathbf{A}=(2 \pi)^{-3 / 2} 2 \Re \iint u_{\mathbf{p}_{\nu}}^{*}(\mathbf{q}-\mathbf{p})(\hat{\mathbf{A}}(\mathbf{p}) \cdot \boldsymbol{\sigma})\left(\boldsymbol{\omega}_{\mathbf{q}} \cdot \boldsymbol{\sigma}\right) u_{\mathbf{p}_{\nu}}(\mathbf{q}) d \mathbf{p} d \mathbf{q} \\
=(2 \pi)^{-3 / 2} 2 \Re \iint\left[\hat{\mathbf{A}}(\mathbf{q}-\mathbf{p}) \cdot \boldsymbol{\omega}_{\mathbf{q}} u_{\mathbf{p}_{\nu}}^{*}(\mathbf{p}) u_{\mathbf{p}_{\nu}}(\mathbf{q})\right. \\
\left.+i u_{\mathbf{p}_{\nu}}^{*}(\mathbf{p})\left(\hat{\mathbf{A}}(\mathbf{q}-\mathbf{p}) \times \boldsymbol{\omega}_{\mathbf{q}}\right) \cdot \boldsymbol{\sigma} u_{\mathbf{p}_{\nu}}(\mathbf{q})\right] d \mathbf{p} d \mathbf{q} .
\end{aligned}
$$

We first observe that

$$
\begin{aligned}
& 2 \Re \iint\left[i u_{\mathbf{p}_{\nu}}^{*}(\mathbf{p})\left(\hat{\mathbf{A}}(\mathbf{q}-\mathbf{p}) \times \boldsymbol{\omega}_{\mathbf{q}}\right) \cdot \boldsymbol{\sigma} u_{\mathbf{p}_{\nu}}(\mathbf{q})\right] d \mathbf{p} d \mathbf{q} \\
& \quad=2 \Re \iint\left[i u_{\mathbf{p}_{\nu}}^{*}(\mathbf{p})\left(\hat{\mathbf{A}}(\mathbf{q}-\mathbf{p}) \times \boldsymbol{\omega}_{\mathbf{q}}\right) \cdot \mathbf{n}_{2} \sigma_{2} u_{\mathbf{p}_{\nu}}(\mathbf{q})\right] d \mathbf{p} d \mathbf{q}=0 .
\end{aligned}
$$

The terms containing $\sigma_{1}$ and $\sigma_{3}$ vanish as they are clearly imaginary. The term with $\sigma_{2}$ vanishes beacuse of the choice (19) of $u_{\mathbf{p}_{\nu}}$.

Note that $u_{\mathbf{p}_{\nu}}^{*}(\mathbf{p}) u_{\mathbf{p}_{\nu}}(\mathbf{q})=0$ unless $|\mathbf{p}-\mathbf{q}|<2 \delta$ and $\left|\mathbf{q}-\mathbf{p}_{\nu}\right| \leq \delta$. Thus $\boldsymbol{\omega}_{\mathbf{p}_{\nu}}-\boldsymbol{\omega}_{\mathbf{q}}=p_{\nu}^{-1}\left(\mathbf{p}_{\nu}-\mathbf{q}\right)+\boldsymbol{\omega}_{\mathbf{q}} p_{\nu}^{-1}\left(q-p_{\nu}\right)$ and we obtain for $\left|\mathbf{p}_{\nu}-\mathbf{q}\right|<2 \delta$ that

$$
\left|\boldsymbol{\omega}_{\mathbf{p}_{\nu}}-\boldsymbol{\omega}_{\mathbf{q}}\right| \leq 2 \delta p_{\nu}^{-1} \leq 2 \delta P^{-1}
$$


where we used that $p_{\nu} \geq P$. Since $\boldsymbol{\omega}_{\mathbf{p}_{\nu}} \cdot \mathbf{n}_{1}>1-\theta^{2}$ we have that $\left|\boldsymbol{\omega}_{\mathbf{p}_{\nu}}-\mathbf{n}_{1}\right| \leq \theta$ and hence

$$
\left|\hat{\mathbf{A}}(\mathbf{q}-\mathbf{p}) \cdot\left(\boldsymbol{\omega}_{\mathbf{q}}-\mathbf{n}_{1}\right)\right| \leq\left(2 \delta P^{-1}+\theta\right) A_{0} \leq 2 \theta A_{0} .
$$

Hence, since $|\mathbf{p}-\mathbf{q}|<2 \delta$ we get from (18) that

$$
\hat{\mathbf{A}}(\mathbf{q}-\mathbf{p}) \cdot \boldsymbol{\omega}_{\mathbf{q}} \leq-A_{0}\left[\left(\boldsymbol{\omega}_{\mathbf{q}-\mathbf{p}} \cdot \mathbf{n}_{2}\right)^{2}-2 \theta\right] .
$$

Thus,

$$
\begin{aligned}
\int \mathbf{j}_{\nu} \cdot \mathbf{A} & =(2 \pi)^{-3 / 2} 2 \Re \iint \hat{\mathbf{A}}(\mathbf{q}-\mathbf{p}) \cdot \boldsymbol{\omega}_{\mathbf{q}} u_{\mathbf{p}_{\nu}}^{*}(\mathbf{p}) u_{\mathbf{p}_{\nu}}(\mathbf{q}) d \mathbf{q} d \mathbf{p} \\
& \leq-\frac{3}{(2 \pi)^{5 / 2}} A_{0} \delta^{-3} \iint_{|\mathbf{q}|,|\mathbf{p}|<\delta}\left[\left(\boldsymbol{\omega}_{\mathbf{q}-\mathbf{p}} \cdot \mathbf{n}_{2}\right)^{2}-2 \theta\right] d \mathbf{p} d \mathbf{q} \\
& =\frac{3}{(2 \pi)^{5 / 2}} A_{0} \delta^{3} \iint_{|\mathbf{q}|,|\mathbf{p}|<1}\left[\left(\boldsymbol{\omega}_{\mathbf{q}-\mathbf{p}} \cdot \mathbf{n}_{2}\right)^{2}-2 \theta\right] d \mathbf{p} d \mathbf{q}
\end{aligned}
$$

We now make the choice

$$
\theta=\frac{1}{3}\left(\iint_{|\mathbf{q}|,|\mathbf{p}|<1} 1 d \mathbf{p} d \mathbf{q}\right)^{-1} \iint_{|\mathbf{q}|,|\mathbf{p}|<1}\left(\boldsymbol{\omega}_{\mathbf{q}-\mathbf{p}} \cdot \mathbf{n}_{2}\right)^{2} d \mathbf{p} d \mathbf{q}
$$

and arrive at

$$
\int \mathbf{j}_{\nu} \cdot \mathbf{A} \leq-\frac{4}{3}(2 \pi)^{-1 / 2} A_{0} \theta \delta^{3} .
$$

From (21) and (23) we therefore obtain

$$
\left(\Psi, \sum_{\nu=1}^{N} D_{\nu}(\mathbf{A}) \Psi\right) \leq \sum_{\nu=1}^{N}\left[\left|\mathbf{p}_{\nu}\right|+\delta-\frac{4}{3}(2 \pi)^{-1 / 2} A_{0} e \delta^{3} \theta\right] .
$$


C. The nuclear coordinates. Finally, we show how to choose the nuclear coordinates following an idea in [18]. Consider the electronic density of the state $\Psi, \rho(\mathbf{r})=\sum_{\nu=1}^{N}\left|\psi_{\mathbf{p}_{\nu}}(\mathbf{r})\right|^{2}$ then

$$
\left(\Psi, V_{c} \Psi\right) \leq-\sum_{k=1}^{K} \int \frac{Z_{\kappa} \rho(\mathbf{r})}{\left|\mathbf{r}-\mathbf{R}_{k}\right|} d \mathbf{r}+D(\rho, \rho)+\sum_{\substack{\kappa, \lambda>1 \\ \kappa<\lambda}}^{K} \frac{Z_{\kappa} Z_{\lambda}}{\left|\mathbf{R}_{\kappa}-\mathbf{R}_{\lambda}\right|},
$$

were we introduced $D(\rho, \rho):=\frac{1}{2} \iint \rho(\mathbf{r})\left|\mathbf{r}-\mathbf{r}^{\prime}\right|^{-1} \rho\left(\mathbf{r}^{\prime}\right) d \mathbf{r} d \mathbf{r}^{\prime}$.

Note now that $\int N^{-1} \rho=1$, i.e., $N^{-1} \rho$ can be considered a probability distribution. We may therefore average $\left(\Psi, V_{c} \Psi\right)$ considered as a function of $\mathbf{R}_{1}, \ldots, \mathbf{R}_{K}$ with respect to the probability measure

$$
\mathbf{R}_{1}, \ldots, \mathbf{R}_{K} \mapsto N^{-1} \rho\left(\mathbf{R}_{1}\right) \cdots N^{-1} \rho\left(\mathbf{R}_{K}\right)
$$

We obtain

$$
\begin{aligned}
& \int\left(\Psi, V_{c} \Psi\right) N^{-1} \rho\left(\mathbf{R}_{1}\right) \cdots N^{-1} \rho\left(\mathbf{R}_{K}\right) d \mathbf{R}_{1} \cdots d \mathbf{R}_{K} \\
= & {\left[(1-(Z / N))^{2}-N^{-2} \sum_{\kappa=1}^{K} Z_{\kappa}^{2}\right] D(\rho, \rho) . }
\end{aligned}
$$

We shall prove that $[\ldots]<0$. There are two cases.

1) $N \geq Z$ : By moving electrons to infinity we may assume that $Z \leq N<$ $Z+1$. Therefore $\left[(1-(Z / N))^{2}-N^{-2} \sum_{\kappa=1}^{K} Z_{\kappa}^{2}\right] \leq Z^{-2}-(Z+1)^{-2} \sum_{\kappa=1}^{K} Z_{\kappa}^{2} \leq Z^{-2}-2(Z+1)^{-2}<0$. 
2) $N<Z$ : We may move nuclei to infinity and assume that $N<Z<$ $N+\max _{\kappa} Z_{\kappa}$. Therefore

$$
\left[(1-(Z / N))^{2}-N^{-2} \sum_{\kappa=1}^{K} Z_{\kappa}^{2}\right] \leq\left(\max _{\kappa} Z_{\kappa}\right)^{2} N^{-2}-N^{-2} \sum_{\kappa=1}^{K} Z_{\kappa}^{2} \leq 0 .
$$

We can therefore find nuclear positions $\mathbf{R}_{1}, \ldots, \mathbf{R}_{K}$ such that $\left(\Psi, V_{c} \Psi\right) \leq$ 0.

Using these coordinates together with (17) and (25) we get

$$
\mathcal{E}_{0}[\Psi, \Psi] \leq \sum_{\nu=1}^{N}\left[p_{\nu}+\delta-\frac{4}{3}(2 \pi)^{-1 / 2} A_{0} e \delta^{3} \theta\right]+2^{-1} 5^{4} A_{0}^{2} \delta^{5} .
$$

We now choose $P=\left(\frac{4 N}{7 \theta^{2}}\right)^{1 / 3} \delta$ in accordance with (20). We then choose $A_{0}$ such that $\frac{4}{3}(2 \pi)^{-1 / 2} A_{0} e \delta^{3} \theta=4 P$, i.e.,

$$
A_{0}=\frac{3}{112^{1 / 3}}(2 \pi)^{1 / 2} \theta^{-5 / 3} e^{-1} \delta^{-2} N^{1 / 3}
$$

Then, using Condition 2 we have $p_{\nu}-(8 \pi / 3) A_{0} e \delta^{3} \theta \leq-2 P$. If we insert these values and estimates above we find

$$
\mathcal{E}_{0}[\Psi, \Psi] \leq \delta\left[-2 N^{4 / 3}\left(4 /\left(7 \theta^{2}\right)\right)^{1 / 3}+N+3^{2} 5^{4} 112^{-2 / 3} \pi \theta^{-10 / 3} \alpha^{-1} N^{2 / 3}\right] .
$$

It is now clear that the expression in [] will be negative for $N$ sufficiently large. The energy can therefore be made arbitrarily negative by choosing $\delta$ large. 


\section{A BKS Inequalities}

As a convenience to the reader we give a proof of some cases of the inequalities due to Birman, Koplienko, and Solomyak [2]. The case needed in Section 3 corresponds to $p=2$ below. There we are interested in $(B-A)_{-}$, but here we treat $(B-A)_{+}$to simplify keeping track of signs. The proof is the same. Recall that $X_{+}:=(|X|+X) / 2$.

Theorem 3 Let $p \geq 1$ and suppose that $A$ and $B$ are two nonnegative, selfadjoint linear operators on a separable Hilbert space such that $\left(B^{p}-A^{p}\right)_{+}^{1 / p}$ is trace class. Then $(B-A)_{+}$is also trace class and

$$
\operatorname{tr}(B-A)_{+} \leq \operatorname{tr}\left(B^{p}-A^{p}\right)_{+}^{1 / p}
$$

Proof. Our proof will use essentially only two facts: $X \mapsto X^{-1}$ is operator monotone decreasing on the set of nonnegative self-adjoint operators (i.e., $X \geq Y \geq 0 \Longrightarrow Y^{-1} \geq X^{-1}$ ) and $X \mapsto X^{r}$ is operator monotone increasing on the set of nonnegative self-adjoint operators for all $0<r \leq 1$. Consequently, $X \mapsto X^{-r}$ is operator monotone decreasing for $0<r \leq 1$.

As a preliminary remark, we can suppose that $B \geq A$. To see this, write $B^{p}=A^{p}+D$. If we replace $B$ by $\left[A^{p}+D_{+}\right]^{1 / p}$ then $\left(B^{p}-A^{p}\right)_{+}=D_{+}$is unchanged, while $X:=B-A \mapsto\left[A^{p}+D_{+}\right]^{1 / p}-A$ can only get bigger because 
$X \mapsto X^{1 / p}$ is operator monotone on the set of positive operators. Since the trace is also operator monotone, we can therefore suppose that $D=D_{+}$, i.e., $B^{p}=A^{p}+C^{p}$ with $A, B, C \geq 0$. Our goal is to prove that

$$
\operatorname{tr}\left[\left(A^{p}+C^{p}\right)^{1 / p}-A\right] \leq \operatorname{tr} C
$$

under the assumption that $C$ is trace class.

To prove (26) we consider the operator $X:=\left[A^{p}+C^{p}\right]^{1 / p}-A$, which is well defined on the domain of $A$. We assume, at first, that $A^{p} \geq \varepsilon^{p}$ for some positive number $\varepsilon$. Then, by the functional calculus, and with

$$
E:=\left[A^{p}+C^{p}\right]^{(1-p) / p} \quad \text { and } \quad P:=A^{1-p}-E
$$

we have

$$
X=E\left[A^{p}+C^{p}\right]-A^{1-p} A^{p}=-P A^{p}+E C^{p}
$$

Clearly, $P \geq 0$ and $0 \leq P \leq \varepsilon^{1-p}$.

Let $Y:=E C^{p}$. We claim that $Y$ is trace class. This follows from $Y^{*} Y=C^{p} E^{2} C^{p} \leq C^{p} C^{2-2 p} C^{p}=C^{2}$. Thus, $|Y| \leq C$, and hence $\operatorname{tr} Y=$ $\operatorname{tr} C^{p / 2} E C^{p / 2} \leq \operatorname{tr} C$

It is also true that $P$ is trace class. To see this, use the integral representation, with suitable $c>0, A^{1-p}=c \int_{0}^{\infty}(t+A)^{-1} t^{1-p} d t$. Use this twice and 
then use the resolvent formula. In this way we find that

$$
P=c \int_{0}^{\infty}\left(A^{p}+t\right)^{-1} C^{p}\left(A^{p}+C^{p}+t\right)^{-1} t^{(1-p) / p} d t
$$

Since $C$ is trace class, so is $C^{p}$, and the integral converges because of our assumed lower bound on $A$. Thus, $P$ is trace class and hence there is a complete, orthonormal family of vectors $v_{1}, v_{2}, \ldots$, each of which is an eigenvector of $P$.

Since $X \geq 0$, the trace of $X$ is well defined by $\sum_{j=1}^{\infty}\left(v_{j}, X v_{j}\right)$ for any complete, orthonormal family. The same remark applies to $E C^{p}$ since it is trace class. Thus, to complete the proof of (26) it suffices to prove that $\left(v_{j}, P A^{p} v_{j}\right) \geq 0$ for each $j$. But this number is $\lambda_{j}\left(v_{j}, A^{p} v_{j}\right) \geq 0$, where $\lambda_{j}$ is the (nonnegative) eigenvalue of $P$, and the positivity follows from the positivity of $A$.

We now turn to the case of general $A \geq 0$. We can apply the above proof to the operator $A+\varepsilon$ for some positive number $\epsilon$. Thus we have

$$
\operatorname{tr}\left[\left[(A+\varepsilon)^{p}+C^{p}\right]^{1 / p}-(A+\varepsilon)\right] \leq \operatorname{tr} C
$$

Let $\varphi_{1}, \varphi_{2}, \ldots$ be an orthonormal basis chosen from the domain of $A^{p}$. This basis then also belongs to the domain of $A$ and the domain of $\left[(A+\varepsilon)^{p}+C^{p}\right]^{1 / p}$ 
for all $\varepsilon \geq 0$. We then have

$$
\operatorname{tr} X=\sum_{j}\left(\varphi_{j}, X \varphi_{j}\right)
$$

Note that a-priori we do not know that the trace is finite, but since the operator is non-negative this definition of the trace is meaningful. Operator monotonicity of $X^{1 / p}$ gives

$$
\left(\varphi_{j},\left[\left[(A+\varepsilon)^{p}+C^{p}\right]^{1 / p}-(A+\varepsilon)\right] \varphi_{j}\right) \geq\left(\varphi_{j},(X-\varepsilon) \varphi_{j}\right)
$$

It therefore follows from (28), followed by Fatou's Lemma applied to sums that

$$
\begin{aligned}
\operatorname{tr} C & \geq \liminf _{\varepsilon \rightarrow 0} \sum_{j}\left(\varphi_{j},\left[\left[(A+\varepsilon)^{p}+C^{p}\right]^{1 / p}-(A+\varepsilon)\right] \varphi_{j}\right) \\
& \geq \sum_{j} \liminf _{\varepsilon \rightarrow 0}\left(\varphi_{j},\left[\left[(A+\varepsilon)^{p}+C^{p}\right]^{1 / p}-(A+\varepsilon)\right] \varphi_{j}\right) \\
& \geq \operatorname{tr} X .
\end{aligned}
$$

\section{B Counting Spin States}

Our goal here is to prove that when $\Psi$ is in $\mathcal{H}_{N, \mathcal{A}}$, the antisymmetric tensor product of the positive energy subspace of the Dirac operator (with or without a magnetic field, $\mathcal{A}$ ) then the one-body density matrix is bounded by 2 
and not merely by 4 , as would be the case if there were no restriction to the positive energy subspace. This result will allow us to use 2 instead of 4 in inequalities (6) and (14). We thank Michael Loss for the idea of this proof.

The one-body density matrix is defined in terms of an $N$-body density matrix (or function) by the partial trace over $N-1$ variables. We illustrate this for functions, but the proof works generally. If $\Psi$ is a function, then $\Gamma\left(\mathbf{r}, \sigma ; \mathbf{r}^{\prime}, \sigma^{\prime}\right):=N \int_{(N-1)} \Psi\left(\mathbf{r}, \sigma, z_{2}, z_{3}, \ldots, z_{N}\right) \overline{\Psi\left(\mathbf{r}^{\prime}, \sigma^{\prime}, z_{2}, \ldots, z_{N}\right)} d z_{2} \cdots d z_{N}$, where $z$ denotes a pair $\mathbf{r}, \sigma$ and $d z$ denotes integration over $\mathbb{R}^{3}$ and summation over the $q$ 'spin' states of $\sigma$. We are interested in $q=4$, but that is immaterial for the definition.

The kernel $\Gamma$ is trace class; in fact its trace is $q N$. It is also obviously positive definite as an operator. The first remark is that $\Gamma \leq 1$ as an operator. To prove this easily, let $\psi$ be any normalized function of one space-spin variable $z$ and define the function of $N+1$ variables $\Phi\left(z_{0}, \ldots, z_{N}\right):=\psi\left(z_{0}\right) \Psi\left(z_{1}, \ldots, z_{N}\right)+\sum_{j=1}^{N}(-1)^{j} \psi\left(z_{j}\right) \Psi\left(z_{0}, \ldots, \hat{z}_{j}, \ldots, z_{N}\right)$, where $\hat{z}_{j}$ denotes the absence of $z_{j}$. This function $\Phi$ is clearly antisymmetric and the integral over all variables of its square is surely nonnegative. However, this integral is easily computed (using the normalization of $\psi$ and $\Psi$ ) to be $(N+1)-(N+1)(\psi, \gamma \psi)$. 
The next step is to consider the reduced kernel (without spin) defined by

$$
\gamma\left(\mathbf{r}, \mathbf{r}^{\prime}\right):=\sum_{\sigma=1}^{q} \Gamma\left(\mathbf{r}, \sigma ; \mathbf{r}^{\prime}, \sigma\right),
$$

which evidently satisfies the operator inequality $0 \leq \gamma \leq q$, since $\Gamma \leq 1$.

The electron density referred to in Section 4.1 is defined by

$$
\rho(\mathbf{r}):=\gamma(\mathbf{r}, \mathbf{r})
$$

but it will not be needed in this Appendix. Another quantity of interest is the current, defined by

$$
\mathbf{j}(\mathbf{r}):=\sum_{\sigma, \tau} \Gamma(\mathbf{r}, \sigma ; \mathbf{r}, \tau) \boldsymbol{\alpha}_{\sigma, \tau}
$$

It follows from this that $|\mathbf{j}(\mathbf{r})| \leq \rho(\mathbf{r})$ for every $\mathbf{r} \in \mathbb{R}^{3}$.

Our goal here is to prove the following fact about $\gamma$ :

If the $N$-body $\Psi$ is in $\mathcal{H}_{N, \mathcal{A}}$ then the corresponding $\gamma$ satisfies $0 \leq \gamma \leq 2$ as an operator.

To prove this we introduce the unitary matrix in spin-space (related to the charge conjugation operator)

$$
U=\left(\begin{array}{cc}
0 & 1 \\
-1 & 0
\end{array}\right)
$$

where 1 denotes the unit $2 \times 2$ unit matrix. With a slight abuse of notation, we shall also use $U$ to denote the $U \otimes 1$ acting on the full one-particle space, i.e., 
$(U)\left(\mathbf{r}, \sigma^{\prime}\right)=\sum_{\sigma} U\left(\sigma^{\prime}, \sigma\right) f(\mathbf{r}, \sigma)$. The important point to note, and which is easily verified from the Dirac equation, is that $\psi \in \mathcal{H}_{+}$if and only if $U \psi \in \mathcal{H}_{-}$, the negative spectral subspace of $D(\mathcal{A})$.

Given $f \in L^{2}\left(\mathbb{R}^{3}\right)$, we define $F^{\tau}$ to be the spinor $F^{\tau}(\mathbf{r}, \sigma):=f(\mathbf{r}) \delta_{\sigma, \tau}$. Then evidently $(f, \gamma f)=\sum_{\tau}\left(F^{\tau}, \Gamma F^{\tau}\right)$. However, since the matrix $U$ merely permutes the spin indices and possibly changes the sign from + to - , we have that $\sum_{\tau}\left(F^{\tau}, \Gamma F^{\tau}\right)=\sum_{\tau}\left(F^{\tau}, \Gamma_{U} F^{\tau}\right)$, with $\Gamma_{U}:=U^{-1} \Gamma U$. (Actually, the proof only requires that $U$ be unitary, nothing more.)

We claim that $\Gamma+\Gamma_{U} \leq 1$ in which case we have proved that $(f, \gamma f) \leq$ $q / 2=2$, as claimed. To see this, we note that $\Gamma \leq 1$ on $\mathcal{H}_{+}$and $\Gamma_{U} \leq 1$ on $\mathcal{H}_{-}$. Since the two subspaces are orthogonal, $\Gamma+\Gamma_{U} \leq 1$ on the whole spinor space.

ACKnowledgment: The authors thank Michael Loss for valuable discussions, especially with regard to Appendix B. After we had proved the results in this paper, including the inequalities in Appendix A, Huzihiro Araki kindly informed us of the paper by Birman, Koplienko, and Solomyak [2] in which the inequalities of Appendix A were proved 21 years earlier; we are grateful to him for this help. We are also greatful to M. Griesemer for pointing out several errors in the preliminary version of this paper. - The authors also thank the following organizations for 
their support: the Danish Science Foundation, the European Union, TMR grant FMRX-CT 96-0001, the U.S. National Science Foundation, grant PHY95-13072, and NATO, grant CRG96011.

\section{References}

[1] H. A. Bethe and E. E. Salpeter. Quantum mechanics of one- and twoelectron atoms. In S. Flügge, editor, Handbuch der Physik, XXXV, pages 88-436. Springer, Berlin, 1 edition, 1957.

[2] M. S. Birman, L. S. Koplienko, and M. Z. Solomyak. Estimates for the spectrum of the difference between fractional powers of two self-adjoint operators. Soviet Mathematics, 19(3):1-6, 1975. Translation of Izvestija vyssich.

[3] G. Brown and D. Ravenhall. On the interaction of two electrons. Proc. Roy. Soc. London A, 208(A 1095):552-559, September 1951.

[4] L. Bugliaro, J. Fröhlich, G. M. Graf, J.. Stubbe, and C. Fefferman. A Lieb-Thirring bound for a magnetic Pauli Hamiltonian. Preprint, ETHTH/96-31(x):x, x 1996. 
[5] J. G. Conlon. The ground state energy of a classical gas. Commun. Math. Phys, 94(4):439-458, August 1984.

[6] I. Daubechies. An uncertainty principle for Fermions with generalized kinetic energy. Commun. Math. Phys., 90:511-520, September 1983.

[7] F. J. Dyson and A. Lenard. Stability of matter I. J. Math. Phys., 8:423-434, 1967.

[8] F. J. Dyson and A. Lenard. Stability of matter II. J. Math. Phys., 9:698-711, 1967.

[9] W. D. Evans, P. Perry, and H. Siedentop. The spectrum of relativistic one-electron atoms according to Bethe and Salpeter. Commun. Math. Phys., 178:733-746, July 1996.

[10] P. Federbush. A new approach to the stability of matter problem. I. J. Math. Phys., 16:347-351, 1975.

[11] C. Fefferman. On electrons and nuclei in a magnetic field. Texas Math. Phys. Preprint server, 96-376(x):x, x 1996. 
[12] C. Fefferman, J. Fröhlich, and G. M. Graf. Stability of ultraviolet-cutoff quantum electrodynamics with non-relativistic matter. Texas Math. Phys. Preprint server, 96-379(x):x, x 1996.

[13] J. Fröhlich, E. H. Lieb, and M. Loss. Stability of Coulomb systems with magnetic fields. I: The one-electron atom. Commun. Math. Phys., 104:251-270, 1986.

[14] Y. Ishikawa and K. Koc. Relativistic many-body perturbation theory based on the no-pair Dirac-Coulomb-Breit Hamiltonian: Relativistic correlation energies for the noble-gas sequence through $\operatorname{Rn}(Z=86)$, the group-IIB atoms through $\mathrm{Hg}$, and the ions of Ne isoelectronic sequence. Phys. Rev. A, 50(6):4733-4742, December 1994.

[15] H. J. A. Jensen, K. G. Dyall, T. Saue, and K. Faegri. Jr. Relativistic four-component multiconfigurational self- consistent-field theory for molecules: Formalism. J. Chem. Physics, 104(11):4083-4097, March 1996.

[16] E. H. Lieb. Sharp constants in the Hardy-Littlewood-Sobolev and related inequalities. Annals of Mathematics, 118:349-374, 1983. 
[17] E. H. Lieb. On characteristic exponents in turbulence. Commun. Math. Phys, 92:473-480, 1984.

[18] E. H. Lieb and M. Loss. Stability of Coulomb systems with magnetic fields. II: The many-electron atom and the one-electron molecule. Commun. Math. Phys., 104:271-282, 1986.

[19] E. H. Lieb, M. Loss, and H. Siedentop. Stability of relativistic matter via Thomas-Fermi theory. Helv. Phys. Acta, $\mathrm{x}(\mathrm{x}): \mathrm{x}, \mathrm{x}$ 199x. In press.

[20] E. H. Lieb, M. Loss, and J. P. Solovej. Stability of matter in magnetic fields. Phys. Rev. Lett., 75(6):985-989, August 1995.

[21] E. H. Lieb and W. E. Thirring. Bound for the kinetic energy of Fermions which proves the stability of matter. Phys. Rev. Lett., 35(11):687-689, September 1975. Erratum: Phys. Rev. Lett., 36(16):11116, October 1975.

[22] E. H. Lieb and W. E. Thirring. Inequalities for the moments of the eigenvalues of the Schrödinger Hamiltonian and their relation to Sobolev inequalities. In E. H. Lieb, B. Simon, and A. S. Wightman, editors, Studies in Mathematical Physics: Essays in Honor of Valentine Bargmann. Princeton University Press, Princeton, 1976. 
[23] E. H. Lieb and H.-T. Yau. The stability and instability of relativistic matter. Commun. Math. Phys., 118:177-213, 1988.

[24] J. Sucher. Foundations of the relativistic theory of many-electron atoms. Phys. Rev. A, 22(2):348-362, August 1980.

[25] J. Sucher. Foundations of the relativistic theory of many-electron bound states. International Journal of Quantum Chemistry, 25:3-21, 1984.

[26] J. Sucher. Relativistic many-electron Hamiltonians. Phys. Scripta, $36: 271-281,1987$.

[27] W. Thirring, ed. The Stability of Matter: From Atoms to Stars, Selecta of Elliott H. Lieb, Springer Verlag, Berlin, Heidelberg, New York, 1997. 